\title{
SPECIAL ISSUE: Advanced Materials for Photoelectrochemical Cells
}

\section{Photoelectrochemical synthesis of fuels}

Developing sustainable energy resources is one of the most urgent tasks for human beings since the increasing energy demand is in drastic conflict with the limited global fossil fuel storage. Among various types of sustainable energy resources, solar energy is considered to be promising due to its inexhaustibility, high capacity, and environmental benignancy. Artificial photosynthesis, which can store solar energy and chemically convert water or carbon dioxide into solar fuels, provides a realistic strategy to produce fuels in a sustainable way. However, the production of solar fuels in industrial scale still remains a great challenge which could not be overcome without the further advances in basic science research. Among the various options for solar fuel generation, the semiconductor-based photoelectrochemical (PEC) synthesis is one of the most promising and also the most challenging methods. Therefore, SCIENCE CHINA Materials organizes this hot topic of "Advanced Materials for Photoelectrochemical Cells" to show the recent development and challenges in this field.

From a basic scientific perspective, PEC synthesis of fuels is a material and configuration development problem, as the reaction systems need to capture and convert the solar energy efficiently, reliably and inexpensively. Z. Wang and L. Wang (https://doi.org/10.1007/s40843-0189240-y) review the development of PEC water splitting in the aspect of material exploring, fabrication and characterization methods. In addition to water splitting, Xiong et al. (https://doi.org/10.1007/s40843-017-9151-y) systematically summarize the recent advanced designs for PEC $\mathrm{CO}_{2}$ reduction including the subtle construction of photocathodes and novel PEC setups. The challenges and key factors for the future development of PEC cell for solar fuel production are also discussed in the above two reviews.

In basic principles, the semiconductor-based PEC conversion of solar energy is realized by complex photochemical and electrochemical processes, including the charge carrier generation, migration and reaction. Therefore, the simultaneous promotion of such processes is highly desirable to enhance the performance of semiconductor photocatalysts. In the aspect of charge gen- eration enhancement, Fu et al. (https://doi.org/10.1007/ s40843-018-9222-4) develop a facile sodium borohydride method to prepare rutile $\mathrm{TiO}_{2}$ photocatalyst with controllable $\mathrm{V}_{\mathrm{O}}$ defects, which can not only extend the photoresponse to visible light region, but also increase the separation efficiency of charge carriers. The bandgap narrowing and absorbance extending effects of element doping are also demonstrated by Liu et al. (https://doi. org/10.1007/s40843-018-9234-y). A series of B/N-doped $\mathrm{TiO}_{2}$ photocatalysts with different nitrogen concentrations are obtained and show a significant enhancement in light harvesting and PEC water oxidation.

The construction of different junctions is beneficial for charge separation and transportation. In the work by Zhang et al. (https://doi.org/10.1007/s40843-017-9172-x), the heterojunctions between one-dimensional CdS nanowires and $\mathrm{MoS}_{2}$ nanosheets are fabricated and constructed. Ultrafast transient absorption measurements are used to probe the charge carrier dynamics and gain deeper insight into the mechanism behind the enhanced PEC and photocatalytic performance. Z-scheme is another efficient structure to improve the charge separation efficiency. Sun et al. (https://doi.org/10.1007/s40843-0179170-6) fabricate the Z-scheme type composite of CdS/ $\mathrm{Cd} / \mathrm{S}: \mathrm{TiO}_{2} / \mathrm{TiO}_{2}$ and demonstrate the promotion of charge separation by $\mathrm{Z}$-scheme structure. In addition, $\mathrm{p}$-n junction can also promote charge transfer and reduce charge recombination leading to an enhanced photocatalytic activity. Chen et al. (https://doi.org/10.1007/ s40843-017-9171-9) report for the first time that p-type $\mathrm{Cu}_{3} \mathrm{P}$ coupled with n-type $\mathrm{g}-\mathrm{C}_{3} \mathrm{~N}_{4}$ forms a $\mathrm{p}$-n junction to accelerate charge separation and enhance photocatalytic water splitting. Their work proposes an effective guidance to develop efficient noble-metal-free hybrid photocatalysts by $\mathrm{p}-\mathrm{n}$ junction structure. In the work by Shen et al. (https://doi.org/10.1007/s40843-017-9183-2), the distance for photogenerated charge carriers can be greatly shortened through tuning the size and thickness of hollow $\mathrm{TiO}_{2}$ nanospheres, leading to the improved charge separation efficiency and photocatalytic activity.

In the aspect of charge carrier reaction, Xiao et al. (https://doi.org/10.1007/s40843-017-9155-9) report a no- 
vel and effective method of $\mathrm{Au}$ ion implantation and the following high-temperature annealing process to simultaneously enhance the charge surface reaction kinetics as well as charge separation efficiency on $\mathrm{Fe}_{2} \mathrm{O}_{3}$ photoanode. Mai et al. (https://doi.org/10.1007/s40843017-9199-5) also demonstrate that the $\mathrm{Ti}, \mathrm{C}$ doping and $\mathrm{CoOOH}$ oxygen evolution catalyst loading on $\mathrm{Fe}_{2} \mathrm{O}_{3}$ photoanode can simultaneously accelerate the transportation and reaction of charge carriers, leading to a greatly enhanced PEC water splitting activity. In addition to the enhancement of charge reaction, the tuning of charge reaction pathway is also important for efficient PEC synthesis of fuels. $\mathrm{Ng}$ et al. (https://doi.org/10.1007/ s40843-017-9237-2) employ a dual-electrolyte system of $\mathrm{Na}_{2} \mathrm{~S} / \mathrm{Na}_{2} \mathrm{SO}_{3}$ mixture and $\mathrm{pH}$-neutral $\mathrm{Na}_{2} \mathrm{SO}_{4}$ as the respective electrolyte solutions in the anode and cathode chambers of a water splitting cell, which can suppress the photocorrosion of $\mathrm{CuInS}_{2}-\mathrm{In}_{2} \mathrm{O}_{3}-\mathrm{TiO}_{2}$ nanotube heterostructure and lead the charge carriers to participate in water splitting. Their work paves a new avenue in the design of advanced PEC system consisting of small bandgap yet photochemically unstable catalyst, particularly the sulfide materials.

In summary, these outstanding contributions have been aimed to take full advantage of photogenerated charge carriers in semiconductor photocatalysts, which is the key to realize efficient solar energy utilization. In particular, enhancing the generation, accelerating the transport, and promoting the reaction kinetics of charge carriers are fully considered and demonstrated. Different strategies, including extension of the light absorption range, morphological control, heterojunction construction, and surface reaction promotion, have been proved to be of great significance, leading to improved efficiency of charge carrier utilization and activity of solar fuel production. Both guest editors would like to thank the contributors of this special issue for submitting their high-profile manuscripts as well as the referees for their dedication and responsibility. We also would like to thank the editorial team from SCIENCE CHINA Materials for the possibility to assemble this nice special issue.

Guest editors

Jinlong Gong

Professor, School of Chemical Engineering and Technology, Tianjin University, China Email: jlgong@tju.edu.cn

Yat Li

Associate Professor, Department of Chemistry and Biochemistry, University of California, Santa Cruz, USA Email: yatli@ucsc.edu 